\title{
Dynamic Behaviour Analysis of Linear Rotor-Bearing Systems using the Complex Transfer Matrix Technique
}

\author{
B.B. Maharathi \\ Department of Mechanical Engineering, IGIT, Sarang-759146, Orissa, India
}

(Received 9 June 2004; accepted 22 April 2005)

\begin{abstract}
A complex transfer matrix method is developed in the present work for analysing the steady-state response of linear rotor-bearing systems in the frequency domain. The transfer matrix of the shaft segment is derived by considering the state variables of the shaft in, a continuous system sense, to give the most general formulation. In this analysis, all the influencing parameters of the shaft, disk, and bearings are included. A three-disks-rotorbearings system has been used as the physical model to demonstrate the effectiveness of this matrix formulation for the evaluation of the dynamic characteristics of any rotor-bearing system. In order to establish the accuracy of this technique, experiments are also conducted on the same rotor-disks model under various bearing conditions as used in the numerical analysis. The numerical results evaluated by various researchers are also compared with these experimental results.
\end{abstract}

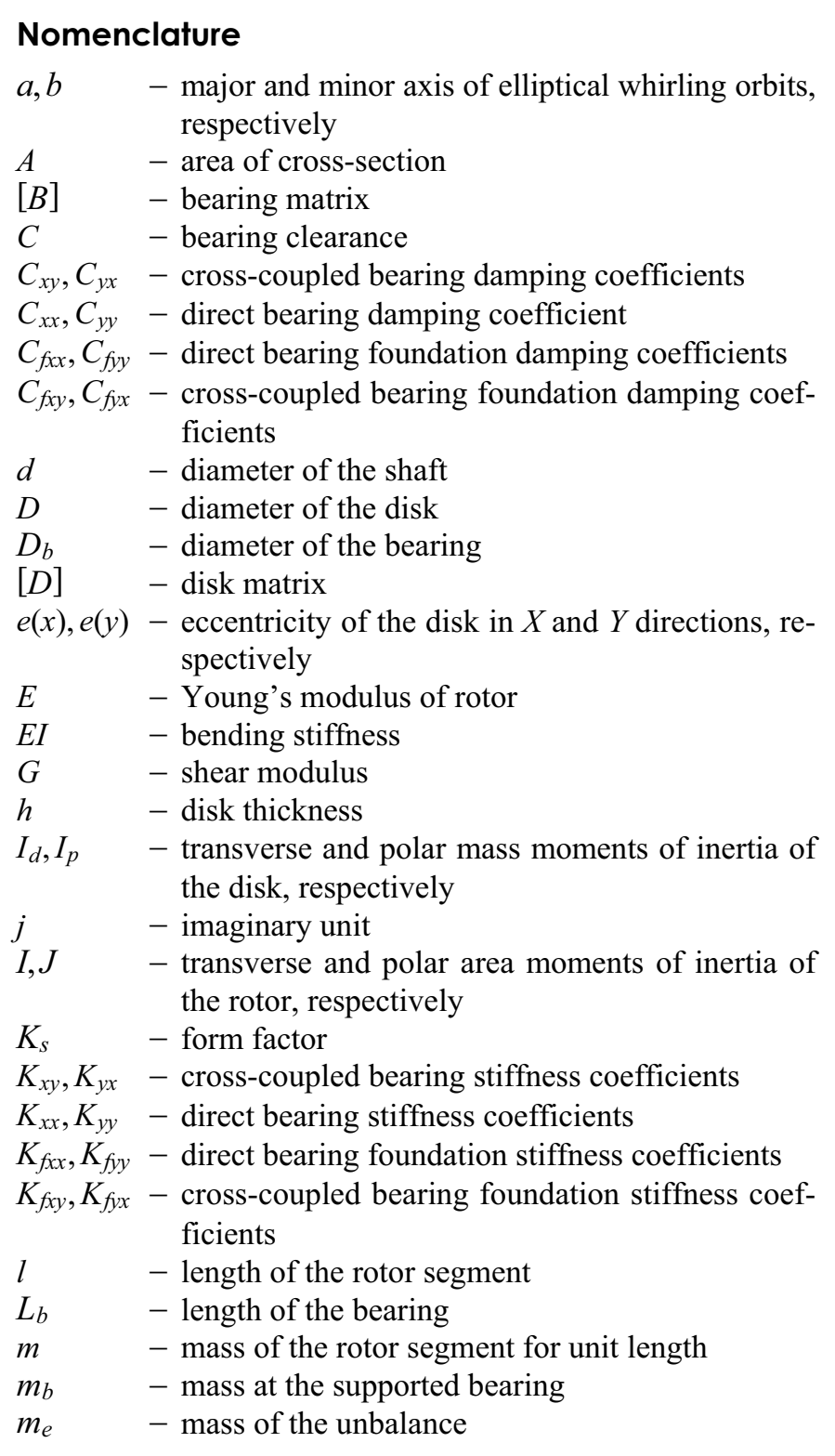

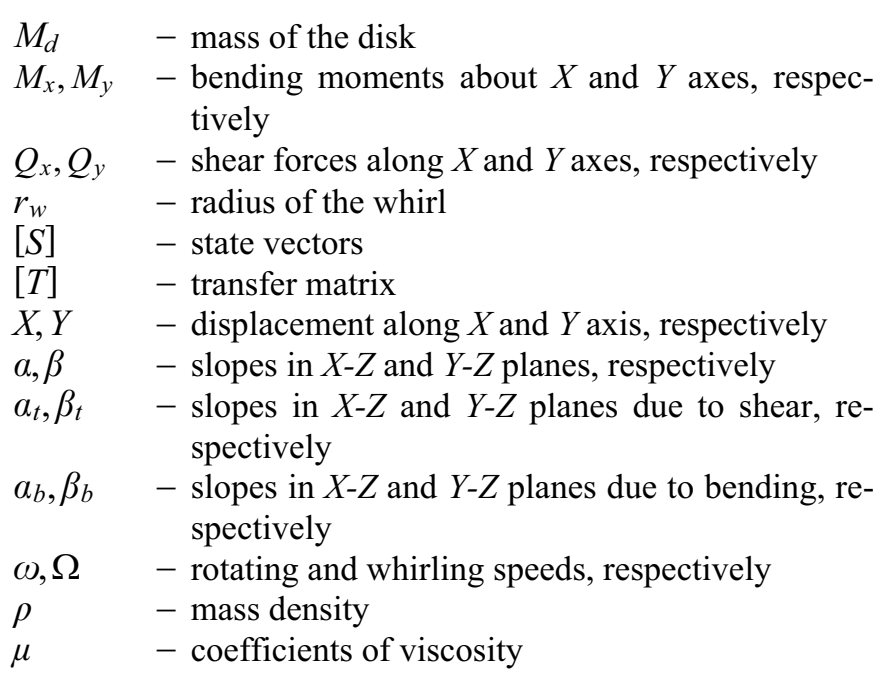

\section{Subscripts}

$C, S$ - associated to cosine and sine terms, respectively

$0, n$ - stage number

\section{Superscripts \\ $L, R-$ left and right to the station, respectively \\ $t \quad-$ transpose of the array}

\section{INTRODUCTION}

The transfer matrix method (TMM) can be used to solve dynamic problems in the frequency domain, which makes it suitable for analysing the steady-state responses of rotorbearing systems with satisfactory accuracy. The TMM was first applied to a rotor-bearing system by Prohl, ${ }^{1}$ who considered rigid bearing characteristics and four state variables. However, subsequently, this concept has been modified by incorporating the dynamic characteristics, such as stiffness and damping coefficients of fluid-film bearings, into the analysis. Kikuchi ${ }^{2}$ and Lund $^{3,4}$ made a significant advancement by including the effects of gyroscopics, internal friction, 